\title{
REMINISZENZEN ZUM LANDSCHAFTSBEGRIFF
}

Im Jahre 1939 stellte der Amerikaner R. Hartshorne in seinem umfänglichen Buch «The Nature of Geography» die These auf, der Landschaftsbegriff sei in Konfusion geraten, weil er in sehr verschiedenem Sinne gebraucht werde. 1927 hatte sein «geistiger Vater» A. Hettner im Werk «Die Geographie, ihre Geschichte, ihr Wesen und ihre Methoden» ungefähr das Gegenteil gesagt. Darin heißt es nämlich : «Die Zusammenstellungen methodischer Äußerungen, wie sie neuerdings mehrfach gemacht worden sind, geben ein falsches Bild von einer Uneinigkeit, wie sie in Wahrheit nicht mehr besteht».

Seit Hettner hätte sich also, nach Hartshorne, die gegenstandstheoretische Situation der Geographie verschlechtert. Eine gewisse Bestätigung dieser Ansicht ließe sich in der Tat auch aus verschiedenen Arbeiten der Jahre 1939-1965 herauslesen. So schließt zum Beispiel die 1958 an dieser Stelle erschienene Dissertation von O. Wernli «Die neuere Entwicklung des Landschaftsbegriffes» unter anderem mit der Empfehlung, diesen Begriff aus der Wissenschaft auszuscheiden, um keine Unklarheiten entstehen zu lassen. Solche Bemerkungen wecken unwillkürlich Vergleiche mit andern Wissenschaften. Warum hat zum Beispiel die Pedologie den Begriff «Boden» nicht längst über Bord geworfen, da er doch in verschiedenen Bedeutungen schillert? Weshalb werden Begriffe wie «Organismus» oder «Gesellschaft», die ebenfalls der Eindeutigkeit ermangeln, nach wie vor und vermutlich auch in der Zukunft weiterhin als Zentralbegriffe der Biologie und Soziologie verwendet, oder warum bedienen sich die jüngst entstandenen Raumwissenschaften (Raumplanung, Raumordnung, Weltraumforschung) ohne weiteres des so fragwürdigen Raumbegriffs, der doch nicht nur sehr verschiedenen Interpretationen unterliegt, sondern vor kurzem auch verhängnisvoll mißbraucht wurde? Oder schließlich: Weshalb entfernen die Geographen den Terminus «Geographie»nicht ebenfalls aus ihrer Wissenschaft, da doch fast jeder Fachgenosse darunter etwas anderes zu verstehen scheint. Als Antwort auf solche Fragen erhält man bei den erwähnten - und andern — Wissenschaften nicht selten die folgende Auslegung: daß es 1. nicht unbedingt darauf ankomme, welche Namen man für einen Gegenstand verwende, sondern $\mathrm{da} B$ es wesentlich sei, wie sie gebraucht werden, da $\beta 2$. auch noch so gut erscheinende Begriffsschöpfungen nie vor «Vervieldeutigungen» geschützt werden könnten und daß es 3. im «Wesen» der Wissenschaft liege, Begriffe immer wieder zu schärfen, zu «präzisieren»; weil damit allein ein Fortschritt möglich sei. $\mathrm{Da} B$ damit nichts gegen notwendige Neuschöpfungen gesagt ist, braucht wohl nicht besonders diskutiert zu werden.

In diesem Lichte gewinnt aber die Kritik am Landschaftsbegriff beziehungsweise die Diskussion um ihn, die in den letzten Jahren erneut aufflammte, vielfach den Charakter eines Streites um Worte, statt um Sachen. Es ist dabei manchmal schwer zu sagen, worum eigentlich gerungen wird. Diesen Eindruck hinterläßt zum Beispiel die interessante Schrift «Der Landschaftsbegriff in der Geographie» von W. Gerling (Würzburg 1965), die sich im Untertitel «Kritik einer Methode» nennt.

Gerling greift in ihr vor allem die Bemühungen an, den Begriff «Landschaft»zum Zentralbegriff der Geographie zu erheben und das dahinterstehende Objekt als «Ganzheit» zu deklarieren. «Die Landschaft als ,Ganzheit' ist ein Scheinproblem», «Der Landschaftsbegriff als, Ganzheit' oder, Gestalteinheit' ist unhaltbar», «Die Personifizierung des Landschaftsbegriffes ist abzulehnen». So heißen Untertitel dieser «Kritik einer Methode». Da es sich um oft diskutierte Fragen handelt, ist man gespannt, welche Gründe Gerling für seine Ablehnung beibringt.

Was zunächst das Ganzheitsproblem (das im Prinzip mit dem Gestalt- und Organismusproblem übereinstimmt) betrifft, beruft er sich auf den «vielzitierten Satz, daß die Landschaft mehr sei als die Summe ihrer Bestandteile». Er betont, daß dieser Satz nicht durch Untersuchung abgeleitet worden sei, sondern eine Behauptung darstelle, «für die bislang der Beweis nicht erbracht worden ist». So wie ihn Gerling zitiert, bedeutet er allerdings wenig, um nicht zu sagen: nichts, da er unterläßt, die denknotwendige Érgänzung - inwiefern denn nun Landschaft mehr sei als Summe - zu geben. Als das «Mehr als die Summe ihrer Bestandteile» hätte sich indessen ohne weiteres die «Summe der Bestandteilsbczichungen» anführen lassen, womit nicht nur die Rechtfertigung der Anwendung des Ganzheitsbegriffs auf die Landschaft gegeben gewesen wäre, sondern sich auch die Behauptung widerlegt hätte, daß der Satz von der «Übersummenhaftigkeit» nicht durch Untersuchung abgeleitet worden sei. Denn ein erheblicher, wenn nicht der größte Teil der bisherigen Geographie hat im Versuch des Nachweises von Beziehungen zwischen den «Landschaftskomponenten» bestanden, was auch Gerling selbst immer wieder unterstreicht, wobei er im «räumlichen Prinzip»die verbind/ende «Klammer» erblickt.

Damit könnte zur «Tagesordnung» übergegangen und sogar Gerlings Satz beigepflichtet werden: «Aus diesem Grunde (weil nämlich das räumliche Prinzip die Teilgebiete der Geographie zum System wie eine Klammer zusammenhalte) bedarf es keiner besonderen zentralen und übergeordneten Kategorie der konstruierten ,Landschaftsganzheit'; keiner besondern ,Gestalteinheit', die wie man sich ausgedrückt hat, das ,Ganze krönen soll'». Aber Gerling hat sich in gewissem Sinne seine Argumentation selbst dadurch verbaut, da $B$ er wiederholt von «Landschaftskomponenten» spricht. Der Begriff Komponente (oder Bestandteil) ist bekanntermaßen 
ein Korrelatbegriff, der den Begriff «Ganzes (Ganzheit), im vorliegenden Fall «Landschaftsganzes», logisch und erkenntniskritisch voraussetzt. Wer letzteren ablehnt, negiert eo ipso die Komponenten, womit natürlich die Geographie überhaupt das Objekt (die Objekte) verlieren würde. Entweder wird demnach auf den Begriff «Komponente» in der Geographie verzichtet (wodurch freilich erhebliche operative Schwierigkeiten entstünden), oder man akzeptiert ihn, womit aber auch das «Ganze»zugleich wieder in sie «hineinkommt».

Darüber, daß es im übrigen beim Zusammenhang der Landschaftskomponenten, der Litho-, Atmo-, Hydro- und Biosphäre, nicht allein um räumliche, sondern um raumzeitliche Probleme geht, wird wohl Gerling sich durchaus klar sein.

Wenn er im gleichen Rahmen außerdem die Forderung erhebt: «Nicht Ganzheitsforschung, sondern Problemforschung ist notwendig», so wird er sich sagen lassen müssen, daß «Ganzheitsforschung» unbestreitbar eines der «problemreichsten Probleme» repräsentiert, die sich einer Wissenschaft stellen. Weder dieser noch andere Sätze - etwa noch: Begriffe wie «Landschaftsganzheit», «Gestalteinheit», «Landschaftsorganismus» sind als theoretische Instrumente geographischer Forschung unbrauchbar, können übrigens als hinreichende Begründung gelten, da sie selbst nur Gegenbehauptungen, nicht aber Beweise darstellen. Ihnen gegenüber haben immerhin so nüchterne Forscher wie I. G. Granö (Geographische Ganzheiten, Peterm. Geogr. Mitteilungen 81, 1935), A. Hettner (Der Begriff der Ganzheit in der Geographie, Geogr. Ztschr. 4o, 1934), E. Plewe (Randbemerkungen zur geographischen Methodik, Geogr.Ztschr. 41, 1935), W. Volz (Geographische Ganzheitlichkeit, Berichte d. mathemat.-physikal. Klasse der sächsischen Akademie der Wissenschaften LXXXIV, 1931) oder R. Wörner (Das geographische Ganzheitsproblem vom Standpunkt der Psychologie, Geogr. Ztschr. 41, 1935) in durchaus kritischer Würdigung der Frage die Verwendbarkeit des Ganzheitsbegriffs für die Charakterisierung erdkundlicher Objekte durchaus bejaht. Sie machten zugleich darauf aufmerksam, daß es verschiedene Ganzheitsbegriffe gibt, von denen sicher nicht alle der Landschaft zugeordnet werden können, was jedoch keinen völligen Ausschluß aus der Geographie präjudiziert. Doch darf hier ohne Wiederholung ihrer Argumente auf sie verwiesen werden.

Ob das im gleichen Zusammenhang von Gerling aufgeworfene «Zentralproblem» der Geographie, die «Integration»der Geosphärenbestandteile (das im Grunde mit dem Ganzheitsproblem identisch ist: Integration = Ganzmachung, d. R.) wirklich nur in seiner Sicht, nämlich im wesentlichen negativ, zu betrachten ist, dürfte ebenfalls diskutabel sein. Gerling schreibt dazu: «Bei der sogenannten ,Integration' der Geosphärenbestandteile darf man sich keiner Täuschung hingeben. Die ,Einheit' der geographischen Substanz ist nur eine vermeintliche, sie ist das Resultat der subjektiven Fähigkeit des Zusammenfügens der vielen räumlichen Objekte bei dem Vorgang menschlicher Wahrnehmung. In Wirklichkeit herrscht eine ungeheuere Mannigfaltigkeit von anorganischen, organischen und auch geistig und seelisch fundierten Erscheinungen, die alle ihren eigenen Ursprung haben und in den Landschaften in immer neuer Weise - gesetzmäßig, regelhaft oder zufällig - zusammentreten. Die Bereiche des Anorganischen, Organischen und Geistigen sind immer durch Sprünge, Grenzen (die aber zugleich Verbindungen darstellen!) und Übergänge getrennt. Auf der Selbständigkeit und Eigenart der anorganischen und organischen Seinsbereiche sowie auf der Besonderheit der geistig-seelischen Vorgänge beruht letzten Endes die grundlegende Einteilung der Wissenschaften (die zumeist die Geographie gar nicht berücksichtigt! d. R.) samt den ihnen adäquaten Verfahrensweisen». Auch diese Thesen setzen Fragezeichen.

Einmal ist «die ungeheuere Mannigfaltigkeit der Erscheinungen»ohne deren gegenseitige Beziehungen weder genetisch noch aktual denkbar. Diese Mannigfaltigkeit ist Ergebnis eines langen Differenzierungsprozesses, der unaufhörlich fortdauert; die «Einzeldinge» haben also keineswegs unbedingt «ihren eigenen Ursprung», sondern entstammen - wie Hettner treffend sagte dem anfänglich «noch nicht differenzierten, wenn man so sagen will: chaotischen Weltall. Dann aber sind sie auch in der Gegenwart nur durch ihre gegenseitigen Kontakte existenzfähig. Man muß also vorsichtig sein, wenn man von ihrer «Selbständigkeit»spricht. Daraufhin deutet übrigens Gerling selbst, wenn er bei ihrer «Trennung» nicht nur «Sprünge», sondern auch «Übergänge» annimmt.

In Wirklichkeit sind indessen bei der Bildung von Einzeldingen und damit auch von Landschaften sowohl Differenzierung wie Integrierung beteiligt, wenn auch deren Anteil (noch) kaum abschätzbar ist. Einheit im Sinne des Zusammenhanges oder der Wechselbeziehungen der Erscheinungen - auch in der Landschaft - kann jedenfalls nicht geleugnet werden. Zusammengefaßt sind Einheit und Vielheit als denknotwendige Korrelatkategorien auch im landschaftlichen Geschehen anzusprechen.

Was schließlich die Vielheit der Wissenschaften anbelangt, darf analog gesagt werden, daß alle Einzeldisziplinen im Grunde Teilbetrachtungen der cincn Gesamtwisscnschaft darstellen. Dies findet im Denkwerkzeug des Geistes und seiner Betätigung ebenso wie in der einen Wirklichkeit, so sehr sie im einzelnen differenziert erscheint, durchaus klaren Ausdruck. Auch die weitere Tatsache, daß alle Einzelwissenschaften mit Analyse und Synthese, Induktion und Deduktion, Empirie und Spekulation, Individualisation und Generalisation usw. zugleich arbeiten 
(müssen), belegt, daß Einheit ebensosehr wie Vielheit sowohl im Gegenstand (in allen Gegenständen) wie im Subjekt (in allen Subjekten) der Wissenschaft leitende Kategorien sind.

Bleiben demnach entschiedene Bedenken gegen eine völlige Ablehnung der Ganzheitserfassung der Landschaft übrig, so muß doch zugegeben werden, daß die hinreichende Abklärung des ganzen, keineswegs einfachen Fragenkomplexes noch aussteht. Sie wird nur auf Grund einer klaren Fixierung des Ganzheitsbegriffes beziehungsweise seiner Varianten selbst und - wie schon Gerling mehrfach betonte - einer Verifikation am konkreten Objekt zu gewinnen sein. Als beherzigenswerter Kern der Gerlingschen Gedanken darf deshalb die Forderung gelten, noch gründlicher und differenzierter als bisher gerade Ordnungsfragen der Geographie — und um solche handelt es sich bei der Konfrontierung der Landschaft mit Ganzheitsbegriffen unbestreitbar-zu behandeln, wobei der Zuzug von Wissenschaftstheoretikern sicher fruchtbar kein könnte.

In der Richtung der Klärung bereits gewirkt zu haben, darf auch J. Schmithüsen für sich in Anspruch nehmen, der kürzlich in zwei Reden die Frage «Landschaft» ebenfalls angeschnitten hat. Sie sind in den Aufsätzen «Der wissenschaftliche Landschaftsbegriff» (Saarbrücken 1963) und «Was ist eine Landschaft» (Wiesbaden 1964) erschienen. Schmithüsen, der eine allgemeine Landschaftskunde vorbereitet, sieht im Gegensatz zu Gerling in der Landschaft nicht nur einen «Grundbegriff» der Geographie. Er erkennt ihm auch Ganzheitlichkeit zu. Auf A. v. Humboldts Umschreibung «Totalcharakter einer Erdgegend»zurückgreifend, stellt auch er zunächst die Vieldeutigkeit des Ausdrucks «Landschaft» fest. Er vertritt aber die Ansicht, daß man diese mittels Präzisierung eines wissenschaftlichen Landschaftsbegriffes überwinden beziehungsweise neutralisieren könne. Die Berechtigung hierzu schöpft er aus dem Bedürfnis nach einer Disziplin, welche das hinter dem Begriff Landschaft stehende Objekt zu erkennen trachtet. Indem er kurz deren Wandel von einer umfassenden Information über die Erde zur Wissenschaft von der Erdhülle (Geosphäre) skizziert, in welcher die «Seinsbereiche des Anorganischen, des Biotischen und des Geistigen in einem System räumlichen (richtiger raumzeitlichen) Zusammenwirkens (Synergismus) vereint» sind, liefert er unmittelbar die durchaus klare und eindeutige Grundlage zur Konzeption eines Landschaftsbegriffs, der tragfähig ist.

Das treibende Motiv sieht er in der Notwendigkeit, die «Geosphäre so zu teilen, daß für eine große $Z$ ahl von Örtlichkeiten gemeinsam gültige Aussagen möglich werden. Man fand die Lösung in der Totalbetrachtung des Zusammenbestehenden im Raum (beziehungsweise im Raumzeitkontinuum, d.R.). Auf Grund vergleichender und anschaulicher Abstraktion erfassen wir einen bestimmten Gestaltcharakter der Geosphäre und nennen seinen Inbegriff die Landschaft (Synergose). Dieser Vorgang ist ähnlich dem, was der Soziologe tut, wenn er eine Gesellschaft in empirisch-deskriptivem Sinne als solche erkannt und von andern unterscheidet, oder der Historiker, wenn er eine Epoche faßt und gegen andere absetzt». Man hat sich dabei zu vergegenwärtigen, daß «man auch an die Konzeption einer Landschaft nicht allzu perfektionistische Ansprüche stellen kann. Diese Einschränkung tut aber dem Wert des Begriffes keinen Abbruch ... Schwierigkeiten liegen vorwiegend in der Theorie... offensichtlich gibt es eine untere Grenze der Größenordnung dessen, was als Landschaft begriffen werden kann (auch eine obere! d. R.). Noch niemand hat es fertig gebracht, diese Grenze zu definieren, obwohl es am konkreten Objekt darüber kaum jemals eine Meinungsverschiedenheit gibt. Wie ein paar Quarzkörner oder ein Feldspatkristall noch kein Granit sind, obwohl sie zu einem solchen gehören, so ist ein Teich, ein Acker oder ein Kirchdorf noch keine Landschaft.

Aber ein in Obstgärten gebettetes Dorf am Rande einer mit Kuhweiden erfüllten Quellmulde, mit Ackerzelgen und ein paar Wegen..., dieses zusammen kann schon die wesentlichsten Züge einer Landschaft ausmachen. Gleichartige Komplexe können sich mit leichter Abwandlung dutzende oder hunderte Male nebeneinander wiederholen. Nach einer gewissen Zahl von Beobachtungen, wenn die Hinzunahme weiterer Proben nichts grundsätzlich Neues mehr einbringt, vermögen wir die Landschaft zu kennzeichnen. Wir können sie dann intensiver studieren nach ihrem substantiellen und formalen Aufbau, nach ihrem Wirkungssystem, ihren Bildungsbedingungen, ihrer Entstehung und nach dem, was zum Beispiel von dem Wesen ihrer Bevölkerung oder dem Nachklang geschichtlicher Vergangenheit in ihr zum Ausdruck kommt.

Wir kennen dann diese Landschaft und können sie verstehen, möglicherweise ohne zu wissen, wie weit sie reicht, wie sie verbreitet ist. «Schmithüsen betont dies, um den Unterschied von Landschaft (Synergose) und Landschaftsraum (Synergochor) deutlich zu machen. Darin liegt insofern eine gewisse Schwierigkeit, als Landschaft selbst schon ein räumliches, genauer: ein sachraumzeitliches Gruppen- oder Komplexgebilde - darstellt. Im Landschaftsraum erblickt Schmithüsen die Voraussetzung der Länderkunde, die in der Regel «Landschaftsysteme höherer Ordnung (Synergeme)»zusammenfaßt. Die hier nur kursorisch wiedergegebenen Gedankengänge führen schließlich zur Definition der Landschaft, welche als «Inbegriff der Beschaffenheit eines auf Grund der Totalbetrachtung als Einheit begreifbaren Geosphärenteils von geographisch relevanter Größenordnung» formuliert wird. Dabei sind offenbar im Begriff «Geosphärenteil» die «Seinsbereiche des Anorganischen, des Biotischen und des Geistigen» schon inbegriffen, ansonst es schwer hielte, die Definition mit den früheren Ausführungen zu koordinieren. Außerdem ist dazu noch zu bemerken, daß der Begriff «Geosphäre»bereits nicht mehr eindeutig gehandhabt wird, so $d a ß$ für seine Verwendung in der Geographie ein präzisierender 
Ausdruck (etwa Gesamtgeosphäre) nötig erscheint. Doch braucht darauf hier nicht eingetreten zu werden.

Es erhebt sich lediglich die Frage, ob aus diesen Gründen eine Bestimmung der Landschaft nicht einfach im Sinne der Assoziation oder des Gefüges von Litho-, Atmo-, Hydro-, Phyto-, Zoound Anthroposphärenteilen und als offenes System - wie dies auch Schmithüsen vorschlägt vorgenommen werden sollte, zumal eine solche auch ohne weiteres verschiedene Landschaftskategorien: Raum- oder Größenkategorien (Lokal-, Regional-, Kontinental-, Globallandschaften usw.), Sachkategorien (Natur-, Kulturlandschaften usw.), Zeitkategorien (A rchaische, mesozoische, antike, mittelalterliche, neuzeitliche, Zukunftslandschaft usw.) oder Funktionskategorien (Progressiv-, Regressiv-, Stagnationslandschaften usw.) zuläßt. $\mathrm{Da} B$ im übrigen «Länder und Landschaften Gegenstände (sind), für die es erst zu erforschen gilt, in welchem Grade sie anorganisch, organisch oder geistbestimmt sind», ist ein Teilproblem, das hier nur gestreift sei. Nichts hindert jedenfalls, daraufhin dem Oberbegriff «Landschaft» anorganische, organische sowie Kulturlandschaften zu subsummieren. Dabei ist dem Ausdruck «belebte Naturlandschaften» mit Schmithüsen den Vorzu zu geben, weil alle Landschaften anorganische Elemente enthalten.

Im Schlußkapitel der anregenden Schrift «Was ist eine Landschaft» zieht Schmithüsen nochmals ein historisches Fazit, bei dem besonders eindrücklich wird, wie weit der Gedanke des Ganzen in Landschaft und Geographie zurückreicht. Von Ritter abgesehen, mutet in diesem Zusammenhang namentlich ein Wort des Königsberger Philosophen K. Rosenkranz (aus dessen «System der Wissenschaft», 1850) sehr modern an: «Landschaften sind relative Ganze, stufenweise integrierte Lokalsysteme von Faktoren aller Naturreiche». Und denjenigen Geographen, der manchmal an seiner Disziplin zweifeln möchte, kann ein Satz des französischen Fachgenossen Vidal de la Blache aufmuntern: Der wesentliche Beitrag der Geographie im Kreise der Wissenschaften sei ihre Fähigkeit, das nicht zu zerstückeln, was in der Wirklichkeit vereint ist.

Mit diesen Zitaten belegt Schmithüsen klar, da $B$ im Objekt der Geographie im Sinne der Landschaftsforschung ein Gebilde zu erkennen versucht wird, das ebensosehr Einheit wie Vielheit verkörpert. Zugleich läßt sich aus seinen Ausführungen ersehen, daß im Grundsätzlichen keinesfalls so viel Unterscheidendes die Geographen trennt, wie oftmals gemeint wird. Denn allen schwebt doch als Objekt die Vergesellschaftung oder Gruppierung der Litho-, Hydro-, Atmo- und Biosphären vor, die sich bald mehr als Zusammengesetztes, bald mehr als geschlossenes Gebilde enthüllt, dessen Elemente aber jedenfalls immer nur im Zusammenhang ihren vollen Sinn empfangen. Damit läßt sich zu der in den Eingangsworten wiedergegebenen Auffassung A. Hettners zurückkehren, die keineswegs Ausruhen auf vollendeter, sondern durchaus Impuls zu vertiefter künftiger Forschung bedeutet.

E. Winkler

\section{Prof. CONSTANTIN VON REGEL, 75jährig}

Am 1o. August 1965 konnte Prof. Dr. Constantin von Regel bei guter Gesundheit seinen fünfundsiebzigsten Geburtstag feiern. C. v. Regel ist, obwohl Botaniker, vielen Geographen durch zahlreiche pflanzengeographische und erdkundliche Arbeiten so bekannt, da $B$ seiner auch an dieser Stelle gedacht werden darf. Er entstammt einem in Gotha beheimateten, 1852 in Zürich eingebürgerten Gelehrtengeschlecht, dessen Haupt, Eduard August Regel, Dr.h.c. der Zürcher Universität, Direktor des kaiserlichen Botanischen Gartens in St. Petersburg, vom Zaren in den erblichen russischen Adelsstand erhoben worden war. C. v. Regel studierte an der Universität St. Petersburg Naturwissenschaften und doktorierte an der Universität Würzburg mit der Dissertation «Die Vegetation der Halbinsel Kola». Sie erschien 1922-27 erweitert als grundlegendes Werk nicht nur der Pflanzengeographie, sondern der Geographie überhaupt. Nach kurzer Privatdozentur in Dorpat wurde Regel 1922 als Ordinarius an die Universität Kaunas gewählt, wo er bis 1940 amtete. Die Rückgliederung Litauens an die UdSSR nötigte ihn zum Verlassen des Landes, und nun begann ein stetes Wanderleben. Es führte zunächst in seine Heimat Schweiz, wo er 1940-43 als Konservator am Herbier Boissier und als Privatdozent an der Universität Genf wirkte. 1944 siedelte er mit seiner Schwester Dorothea v. Regel nach Graz über, von wo aus er verschiedene Forschungsreisen unternahm. Seine zahlreichen Publikationen machten Hochschulen besonders des Nahen Ostens auf ihn aufmerksam. Er erhielt Lehraufträge an den Universitäten Bagdad (1952/55), Istanbul (1956), Kabul (1958/59) und arbeitet seit 1962 als Professor für systematische Botanik an der Universität Izmir, immer bestrebt, die Studenten zu eigenem genauem Beobachten und selbständigem Denken anzuleiten. Die Weite seines Forschens bekunden viele wertvolle Beiträge zur Vegetationskunde der Räume zwischen Weißem und Mittelmeer, Mitteleuropa und Mittelasien. Sie sind auch dem Geographen wertvoll, weil er in ihnen einerseits vielfach Grenzprobleme, anderseits Gebietsgliederungsprobleme angeht. Dabei kam er unmittelbar mit der Geographie selbst in Kontakt, die er ebenfalls mit einer größern Zahl von Untersuchungen bereicherte. Es sei nur an die ältern Arbeiten «Mensch und Wirtschaft in NordRußland» (1922), «die Einteilung Rußlands in natürliche Landschaften» (1930), «die Tundren am Südufer des Weißen Meeres» (1933), «Weißruthenien und seine Bedeutung für Europa» 